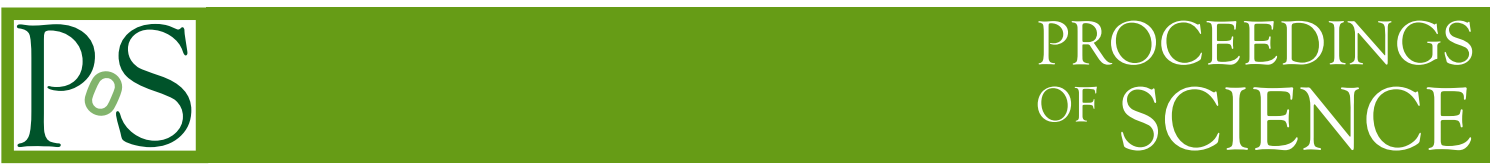

\title{
Close Cathode Chamber, new variant of MWPCs
}

\section{Gergő HAMAR*}

Wigner Research Centre for Physics, Budapest

E-mail: hamar.gergo@wigner.mta.hu

\section{Dezső VARGA}

Wigner Research Centre for Physics, Budapest

E-mail: varga.dezso@wigner.mta.hu

\section{Gábor KISS}

Eötvös Loránd University, Budapest

Wigner Research Centre for Physics, Budapest

E-mail: kiss.gabor@wigner.mta.hu

\begin{abstract}
MWPCs served well for HEP experiments for decades, even with their basic drawbacks, like massive support frames and the need for high precision cathode flatness. Close Cathode Chambers are asymmetric wire chambers, where these constraints are eliminated with appropriate voltages on the alternate anode and field shaping wires. In this configuration the wires can be placed close to the segmented cathode plane, which simplifies the construction, and results in a narrow pad response function. Excellent gain uniformity, average material budget below $2 \%$ radiation length including frames for a chamber with $1 \mathrm{~m}$ long wires, and reduced dead zones are demonstrated. Signal formation is shown to be consistent with classical MWPC-s using advanced simulation techniques. The presentation will focus on the basic concept, details on innovative solutions to fully exploit the advantages, and the applicability as position sensitive detectors in high energyand applied physics experiments.
\end{abstract}

The European Physical Society Conference on High Energy Physics

18-24 July, 2013

Stockholm, Sweden

\footnotetext{
* Speaker.
} 
Multi wire chambers have long excelled for tracking of charged particles, however their construction needs high precision and expertise. Local gain depends strongly on the distance of the wire and the cathode planes, thus requiring high tension on the wires and perfectly flat cathodes; These result massive framing for all these type of detectors, meaning high material budget, heavy lifting, and sensitivity for pressure effects, see e.g. [1].

In "Close Cathode Chambers" (CCC) there are alternating sense- and field wires close to the one of the grounded cathode planes (padplane). With proper voltage ratio on the two types of wires the dependence of gain on the distance from the padplane can be eliminated [2]. As this way cathode flatness and wire sagging does not play a role the whole mechanical structure can be simplified.

Here we report about the experiences gathered during the construction of a $1 \mathrm{~m}$ long, $0.5 \mathrm{~m}$ wide chamber. Within our standards the wire spacing is $4 \mathrm{~mm}$ and their distance from the padplane is $1.5 \mathrm{~mm}$, while the whole gas volume is $10 \mathrm{~mm}$ thick. In this case the voltage ratio should be -0.6 , thus applying roughly $+1000 \mathrm{~V}$ and $-600 \mathrm{~V}$ for sense and field wire respectively (and $-600 \mathrm{~V}$ for the far cathode as well) in $\mathrm{Ar} / \mathrm{CO}_{2}: 80 / 20$ mixture for the desired $>99 \%$ efficiencies.

Laser engraved plastic bars help to keep the wire spacing uniform. We used additional spacers in every $25 \mathrm{~cm}$ allowing the use of even more moderate stretching force. The plastic bars were glued onto the baseplate PCB, which is able to hold the tension of the wires by itself. Several small pillars have been glued between the baseplate and the cathode, making partition from the whole area to reduce the effective force caused by the overpressure inside the chamber. Some pictures from the construction are shown in Fig. 1.

The features of the CCC was proven with calculations and measurements as well [2], however direct comparison with a close to standard MWPC setup was carried out. A large area CCC chamber was used with field wires at CCC and zero (MWPC-like) potentials, sense wire voltage was set to achieve the same gain. Due to the small overpressure of the bubblers the thin chamber pops out, ruining the flatness of the cathode. Gain uniformity with and without overpressure for both CCC and MWPC-like settings are shown in Fig. 2. One can see the effect in MWPC is roughly 30\% while with CCC settings it is well within a $5 \%$ limit.
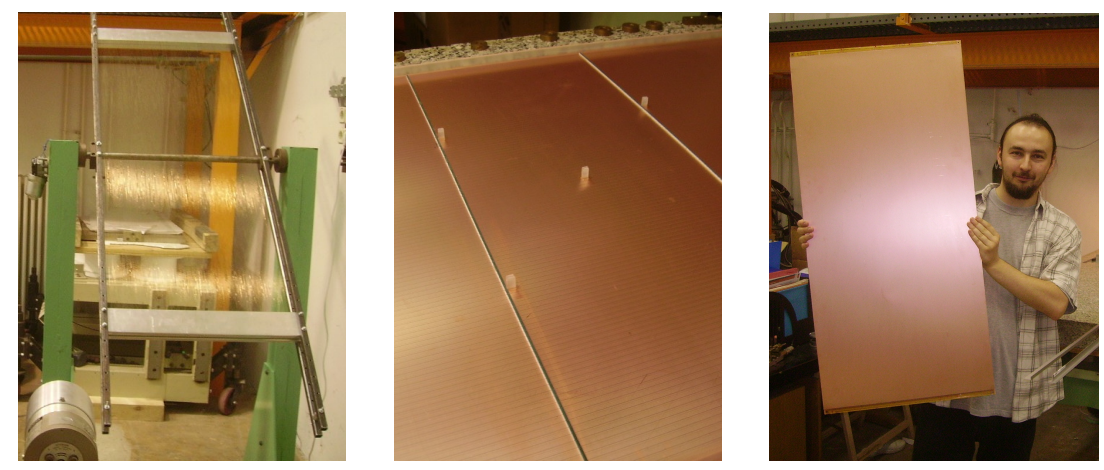

Figure 1: Some of the construction steps of a Close Cathode Chamber: (left) Wires winded onto a temporary frame. (middle) Baseplate with spacers, pillars, and the wires. (right) The chamber with $1 \mathrm{~m}$ long wires is ready. 

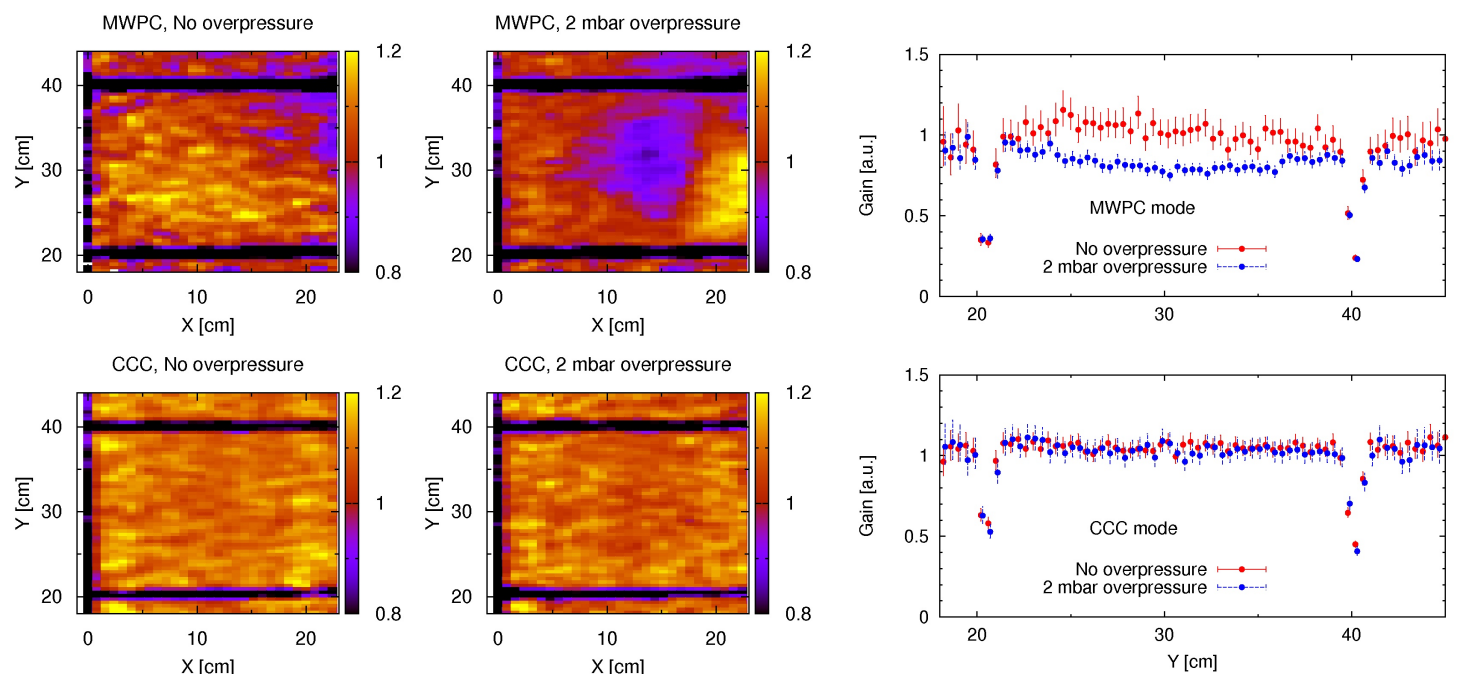

Figure 2: (left) Gain map for CCC and MWPC settings with and without overpressure. (right) One dimensional slice from the figure in the left in the middle of the investigated area.[3]

These features provide the Close Cathode Chambers to be used in high energy physics as low material budget tracking detectors, as it was considered for the tracking and triggering device for a proposed upgrade for LHC ALICE called VHMPID [5].

Their easy construction process and the lightweight structure makes CCC applicable for applied physics, especially for portable detection systems. CCC based cosmic muon tomography experiments are in progress for underground cave/cavity searches [6].

We would like to thank the REGARD, ALICE-VHMPID and ALICE-Budapest groups, and the support of the the Hungarian Momentum ("Lendület") Programme of the HAS, the Hungarian OTKA Grants NK77816, NK106119, and H07-C 74164.
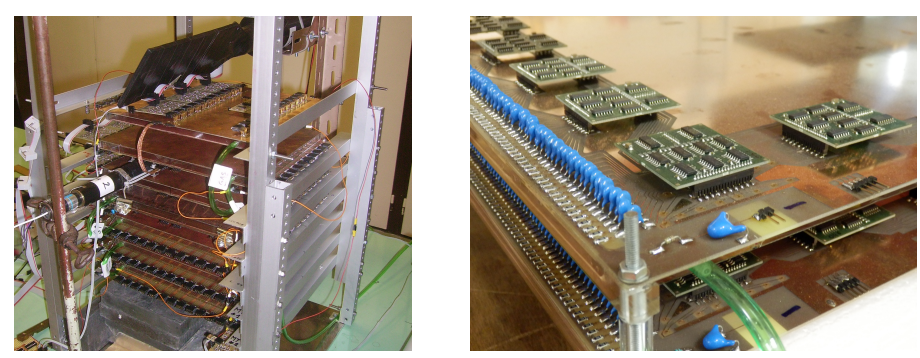

Figure 3: (left) Lightweight cosmic setup from CCC chambers. (right) CCC based detectors with digital electronics connected right underneath the chamber.

\section{References}

[1] G.Charpak, G.Fischer, A.Minten, L.Naumann, F.Sauli, G.Flügge, Ch.Gottfried, R.Tirler, Some Features of Large Muntiwire Proportional Chambers, Nucl. Instr. and Meth. 97 (1971) 377

[2] D.Varga, G.Hamar, G.Kiss, Asymmetric multi-wire proportional chamber with reduced requirements to mechanical precision, Nucl. Instr. and Meth. A 648 (2011) 163

[3] D.Varga, G.Hamar, G.Bencédi, G.Kiss Close Cathode Chamber: Low material budget MWPC, Nucl. Instr. and Meth. A 698 (2013) 11 
[4] F.Sauli, Micro pattern gas detectors, Nucl. Instr. and Meth. A 477 (2002) 1

[5] G.Hamar for the VHMPID Collaboration, VHMPID : ALICE detector upgrade proposal in the high-pT region, CERN Proc. 2012-001 Proc. 6th Int.Ws. High pT Phys. At LHC (2012) 140

[6] G.G.Barnaföldi, D.Varga, L.Oláh, G.Hamar, H.G.Melegh, G.Surányi, Portable Cosmic Muon Telescope for Environmental Applications, Nucl. Instr. and Meth. A 689 (2012) 60 